\title{
Linx
}

Revue des linguistes de l'université Paris X Nanterre

$50 \mid 2004$

Variation sémantique et syntaxique des unités

lexicales : étude de six verbes français

\section{Quelques aspects de commencer}

Rémi Camus

\section{(2) OpenEdition \\ 1 Journals}

Édition électronique

URL : http://journals.openedition.org/linx/139

DOI : 10.4000/linx.139

ISSN : 2118-9692

Éditeur

Presses universitaires de Paris Nanterre

\section{Édition imprimée}

Date de publication : 1 juin 2004

Pagination : 81-101

ISSN : 0246-8743

\section{Référence électronique}

Rémi Camus, "Quelques aspects de commencer», Linx [En ligne], 50 | 2004, mis en ligne le 27 janvier 2011, consulté le 01 mai 2019. URL : http://journals.openedition.org/linx/139; DOI : 10.4000/linx.139 


\title{
Quelques aspects de commencer
}

\author{
Rémi Camus \\ Université de Caen \\ UMR 7110 (Université Paris 7)
}

\section{Avertissement}

Le présent texte résulte d'un parti-pris qui nécessite une brève explication.

Commencer est devenu, notamment à la faveur de l'ouvrage de G. Kleiber (Kleiber, 1999), un banc d'essai pour théories sémantiques. D'un article ayant pour objectif de revisiter ce verbe, le lecteur avisé serait donc en droit d'attendre l'exposé d'une théorie sémantique concurrente, ou au moins une réflexion sur les théories en lice.

Or ce qui a motivé le présent article est justement l'impression que ces enjeux généraux, même s'ils apportaient des pièces cruciales pour l'analyse de commencer, dépassaient à ce point leur objet d'étude qu'ils finissaient par le manquer. Pour le dire sans détour : des pans entiers et, selon moi, essentiels du fonctionnement de commencer se trouvent escamotés: soit parce que les analyses portent exclusivement sur des emplois comparables à ceux de l'anglais begin ${ }^{1}$, soit encore parce que les phénomènes décrits sont liés à des entourages lexicaux et syntaxiques surdéterminés.

Trois grands thèmes sont associés à l'étude du lexème commencer:

\section{- L'aspect}

Sous cette rubrique, le verbe commencer a droit de cité dans toutes les grammaires générales du français, mais seulement dans sa fonction d'auxiliaire. D’où, par un glissement indu, une pétition de principe : ne ressortiraient à la catégorie de

\footnotetext{
${ }^{1}$ Or il se trouve que la relation de traduction entre ces deux termes est complexe (cf. angl. to begin, to start, to commence et leur combinatoire avec on, in, up etc.). Il se trouve également que les étymons respectifs sont clairement disjoints, opposant " aller dans » (commencer < cum-initiare < initium « début, commencement», supin de in-ire «aller dans, entrer») et " engendrer» (be-gin < i.-e. * genb1 $^{2}$ vraisemblablement causatif (L.I.V., s.v. et Remarque 2 p. 64), cf. lat. genuī « engendré », même racine

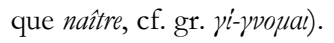


l'aspect que les emplois construits avec à/de + infinitif ou susceptibles de l'être ${ }^{2}$. Le morcellement engendré par l'application de ce critère distributionnel bloque a priori toute tentative d'articulation aspect, détermination et syntaxe. Or les phénomènes paraissent inextricablement liés; mentionnons la distribution différenciée des prédéterminants dans Jean commence un / le / * du fromage (Godard et Jayez, 1993) et Jean commence une / *la grippe; ou encore les interprétations très différentes de la danse dans La danse commence et Ma fille commence la danse.

\section{- La question de l'ellipse}

Il s'agit ici du «surplus de sens» apparemment engendré par l'expression commencer $+S N$ : commencer le fromage s'interprétant comme commencer à manger, confectionner etc. le fromage, commencer un livre s'interprétant comme commencer à lire, rédiger, illustrer... un livre. Ce « surplus » provient-il d'un prédicat enfoui (thèse de l'ellipse), ou bien du forçage de la sémantique des SN un livre, le fromage, rendus processifs (thèse alternative dite de "coerc[it]ion de type », s'inspirant des travaux de J. Pustejovsky)?

La mention de commencer dans les débats autour du concept d'ellipse est relativement ancienne. Dans son article «De l'ellipse» (1975), Claire BlancheBenveniste s'appliquait à montrer que l'analyse par «ellipse du mot adéquat» opposée à «l'ellipse grammaticale » portant sur des structures entières - permet «de régulariser des constructions syntaxiques apparemment disparates ». Un des exemples choisis porte précisément sur trois constructions syntaxiques de commencer:

«Soit trois constructions du verbe commencer, avec trois types de compléments : zéro, un nom, un infinitif précédé de $\grave{a}$ :

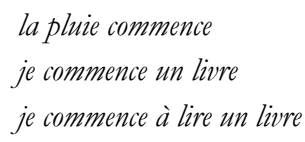

Dans toutes les séquences 1), on peut restituer un verbe adéquat (ici tomber). Dans toutes les séquences 2), on peut restituer également un verbe adéquat (lire, écrire, fabriquer...); le nom est complément de ce verbe, et non de commencer. Si l'on admet cette analyse les trois constructions n'en font qu'une :

commencer $[+\grave{a}+$ verbe $( \pm$ nom $)]$.

Il y a effacement du verbe, en tant que mot adéquat, quand le sujet de commencer

\footnotetext{
${ }^{2}$ B. Peeters, dont les recherches récentes portent sur le lexème commencer en soi (cf. bibliographie), paraît prendre ce point pour acquis. Je cite Peeters (2002), au sujet de la séquence un cimetière commencé l'an dernier: «Puisque le cimetière existait dans sa totalité dès qu'on y a enterré le premier mort, et que Frath signale qu'il est difficile de suppléer un infinitif non exprimé (...), il faut peut-être opter pour une autre analyse, qui rapprocherait le verbe commencer de verbes tels que, par exemple, ouvrir ou inaugurer, plutôt que d'y voir un verbe aspectuel». Faut-il dès lors parler d'un «autre verbe »? La thèse de l'homonymie parait douteuse, d'autres langues attestant un continuum de valeurs analogue. Par exemple, en chinois mandarin, kail (開, caractère représentant deux mains retirant la barre verrouillant une porte) correspond, entre autres traductions, à fr. «ouvrir» (la porte : kai le men), « commencer + SN / + inf. » (kai shi $\left.i^{3}\right)$, « inaugurer » (kai -shet) (Ošanin et al., s.v.).
} 
fournit les indications nécessaires (jugées nécessaires par rapport à un type d'institutionnalisation) pour sa probabilité d'occurrence. » (Cl. Blanche-Benveniste, 1975, p. 39. Tiré à part avec corrections de l'auteur).

Certes, on a montré depuis lors que la mise en œuvre de cette analyse était souvent hasardeuse pour l'étude de commencer (restaurer ou insérer le "mot adéquat» ne va pas de soi) et qu'elle ne rendait pas compte des propriétés en partie divergentes de (2) et (3) (Kleiber, op. cit.). Ajoutons que l'exemple la pluie commence choisi pour illustrer (1) est un hapax lexico-syntaxique : c'est apparemment l'unique exemple où le tour intransitif puisse être étoffé à l'aide du «mot adéquat», en l'occurrence : $[a]$ tomber. Nul infinitif ad hoc ne se laisse rétablir dans Les vacances commencent [?à + infinitif] ; a contrario, l'infinitive ne saurait être supprimée dans La neige commence à tomber ou Le vent commence à souffler.

Néanmoins - et c'est l'essentiel ici - des versions plus prudentes de l'analyse par ellipse restent envisageables et demeurent d'actualité (celles que G. Kleiber dénomme "versions faibles ») ; l'astuce consiste à insérer un procès sous-spécifié sans réalisation phonologique qui compenserait l'absence de dimension événementielle des $\mathrm{SN}$ tels que le livre dans commencer le livre. Moyennant cette mise à jour de la problématique de l'ellipse, le texte cité exprime clairement l'objectif de l' «analyse par ellipse » appliquée à commencer: l'entreprise vise la réduction maximale des structures syntaxiques attestées. Il en va de même, mutatis mutandis, dans la théorie dite de « coerc[it]ion de type » permettant in fine d'assimiler un livre à la lecture d'un livre, ou dans celle de la « supplétion» (cf. Peeters, 2003, qui réduit « huit cadres syntaxiques à trois formules »).

\section{- Les types de procès}

On tient généralement pour acquis que commencer « sélectionne un événement» (Kleiber, op. cit.), ce qui conduit tantôt à nier l'existence d'énoncés où rien ne se passe (Le No12 de la Rue de la Gare commence la Rue Pierre Girard), tantôt à réintroduire après coup le point de vue cursif d'un observateur (Le livre $V$ commence page 8 / par une coquille), tantôt enfin à négliger telle classe d'emplois (Dominique commence le foot, l'hébreu, une vilaine angine, une psychanalyse, la Nivaquine).

S’il y avait sélection d'un événement, comment expliquer également que dans son fonctionnement auxilié, commencer se comporte comme un véritable « receveur universel », acceptant tous les infinitifs imaginables (cf. Frantext et moteurs de recherche sur l'Internet) : commencer à (de) filer, battre, réussir, sentir, toucher, être désagréable, arriver, connaître, contenir... D'ailleurs, l'analyse de Franckel (1989) suggère que l'événementialité n'est pas une condition, mais bien l'un des effets que peut engendrer commencer: c'est bien commencer qui induit l'idée d'une escalade vers un climax dans Je commence à en avoir(vraiment) marre / a être (complètement) fatigué.

A propos de ces trois catégories - aspect, ellipse et types de procès -, commencer est donc convoqué pour défendre et illustrer des théories générales. L’absence de 
discussion détaillée avec ces théories ne marque ici ni un credo réaliste (qui oserait ôter les microscopes en décrétant qu'on voit mieux sans eux ?!), ni une forme aiguë de nonchalance scientifique. Il découle du souhait de recentrer l'étude de commencer sur précisément - le lexème commencer, et ce dans une double optique :

- la question de l'identité des unités de langue ;

- la construction de valeurs référentielles ${ }^{3}$.

Il fallait néanmoins, et dans un espace limité, situer ce travail par rapport aux descriptions existantes. La matière traitée fut donc réduite, et l'accent mis sur certains problèmes autorisant une comparaison des approches. Au risque d'allonger plus que de coutume l'entrée en matière, et au détriment de l'analyse systématique. De celle-ci ne furent retenues que certaines observations inédites destinées à faciliter la confrontation, si tant est que les théories puissent aussi se mesurer à l'aune des phénomènes qu'elles rendent visibles.

\section{Un verbe sans qualité ?}

1.1. Commencer n'évoque pas d'entités particulières, au sens où compter évoque les moutons; tourner, la Terre; ou encore filer, la laine. Certes, le jour, le match, les ennuis sont de bons candidats pour un commencement, mais aucun de ces syntagmes nominaux ne peut prétendre entrer à ce point en résonance avec commencer qu'il parviendrait à dire de façon tout à la fois idiosyncrasique et exemplaire la quintessence de ce qui commence.

A tout prendre, si combinaison prototypique il y avait, ce serait peut-être la séquence ça commence : représentation minimale d'un existant, donc bien une entité (il faut toujours que commencer se dise de quelque support, contrairement à plewvoir), mais pas assez individuée pour être nommée autrement que par le pronom ça. C'est un des points communs de commencer avec un verbe comme sentir.

Mais commencer n'est pas non plus associable à un phénomène physique ou perceptif privilégié, à condition de dénouer ce qui ressortit au verbe de l'apport des constructions où il s'inscrit.

Soit l'exemple des constructions transitives, souvent citées pour illustrer le filtrage référentiel qu'opérerait commencer: Nous commencons la symphonie. Cette phrase hors contexte privilégie certainement l'activité d'interprétation au détriment de l'audition. Mais la contrainte est corrélative à la présence du sujet syntaxique, d'ailleurs de nature agentive. Une fois la transitivité supprimée (La symphonie commence), cette contrainte s'inverse au profit de l'audition: la symphonie est appréhendée par un auditeur, calé dans un fauteuil du parterre ou lui-même installé derrière un pupitre. Par conséquent, l'opposition «interprétation » vs « audition » n'est pas discriminante pour le verbe en tant que tel.

Le même constat peut être fait à propos de l'opposition d'anthologie «lecture » vs « rédaction» dans l'interprétation de J'ai commencé le chapitre IV. Tantôt le chapitre IV

\footnotetext{
${ }^{3}$ Cette double optique correspond en fait à deux programmes de recherches élaborés au sein de la Théorie des Opérations Prédicatives et Énonciatives, respectivement la théorie des «formes schématiques», et la théorie de la «construction des occurrences». Pour une présentation générale, on se reportera, par exemple, à De Vogüé (1999).
} 
désigne un terme qui n'a pas de statut en dehors de sa visée par l'agent (le chapitre n'est pas encore rédigé), auquel cas il ne peut s'agir que de production / rédaction; tantôt le livre existe indépendamment de cette visée, et détermine alors l'extensité de cette activité particulière de reproduction / déchiffrage - pour autrui ou pour soimême - d'un texte qu'on appelle «lecture». Dans les deux cas, c'est bien d'un commencement qu'il s'agit, la variation interprétative «lecture 》 vs « rédaction» découlant du mode de donation du complément. Une alternative qui n'est pas le propre du lexème commencer comme l'attestent des contrastes identifiés de longue date (notamment par Apresjan, 1974) : dessiner un portrait vs un modèle, ouvrir une fenêtre vs une parenthèse, creuser la terre vs un trou etc.

1.2. Commencer ne permet pas en soi de qualifier le sujet syntaxique de lecteur, auteur ou interprète, et l'on peine à imaginer un sens au lexème ?commenceur, du reste inattesté dans les dictionnaires. Exception faite, peut-être, pour l'unique emploi identifié comme «spécialisé » par les lexicographes : de celui qui, garçon vacher ou simple amateur d'équitation, a bien commencé de nombreux chevaux, on admettra peut-être de dire qu'il est un bon commenceur de chevaux. Il reste qu'on n'entendra pas par là quelque opération qualitativement spécifique et calibrée (comparer avec débourrer un cheval) mais l'intervention d'un premier agent, une action inaugurale déterminante pour le devenir du cheval.

1.3. L'apparente atonie sémantique de commencer rapproche ce verbe de ces unités qu'on dit "grammaticales», de l'article dans le syntagme nominal ou d'une flexion dans le mot: une unité de langue dénuée par elle-même de substance notionnelle (et réfractaire à l'idiomaticité) mais impliquant des notions introduites contextuellement : tout commencement est commencement de. De ce point de vue, l'énoncé Le match commence! est une façon de dire qu'un match se déroule, à l'instar de la phrase nominale Le match! qui peut parfois lui être substituée.

Imaginez également la discussion suivante :

A - Pour moi, un match commence vraiment au coup de gong initial, pas au 2eime round ou au premier sang.

B. - N'empêche qu'il n'a toujours pas commencé : il n'y a personne sur le ring. Et s'il n'a pas commencé effectivement, alors ce n'est pas un match.

Même dans ce cas limite où l'on s'applique à faire comprendre ce que veulent dire commencer vraiment et commencer effectivement, c'est en dernier ressort du match en tant qu'événement que l'on devise : a-t-il lieu (B) ? quels sont ses attributs essentiels (A) ? Le commencement lui-même, apparemment fin et impalpable comme une feuille de papier à cigarette, ne se prête pas à ces chipotages.

1.4. En l'absence d'emplois qu'on qualifierait d'«idiomatiques», les descriptions lexicographiques en sont donc réduites à décliner : 
- les schémas argumentaux: SN (La fête commence), SN0-SN1 (je commence le chapitre IV), SN0-[SN1] (construction dite «absolue » caractérisée par un sujet animé : L'abbé commence);

- les types de compléments prépositionnels: commencer à, pour, par, sur, avec, pour ne prendre que les usages les plus courants en français contemporain ; s'ajoute de dans un registre plus livresque ou en français québécois, et des constructions plus rares, voire obsolètes : Commencez.par sa soeur à répandre son sang (Corneille) ;

- des domaines d'application: le temps ou l'espace (le commencement de la ruée ou le commencement de la rue);

- des notions sémantiques : inchoation, causation, localisation.

Toutes distinctions livrées comme autant de façons de redéployer une idée en même temps abstraite et opaque, le plus souvent exprimée tautologiquement sous la forme du dérivé nominal commencement. Par exemple dans le Petit Robert (1989, s.v.) :

« être à son commencement»

« entrer dans son commencement»

Double circularité : non seulement commencement est défini comme le « fait de commencer », mais en outre entrer dans se retrouve en partie dans l'étymologie du mot (cf. note 1 supra).

On comprend que cette présentation est dictée par la structure de l'article dans l'ouvrage cité : sont mis en vedette les emplois transitifs relevant d'un " faire », en conformité avec la chronologie relative des premières attestations ${ }^{4}$ : "Faire la première partie d'une chose ou d'une série de choses ; faire exister (ce qui est le résultat d'une activité) ; (...) faire d'abord ».

Mais une fois ôté le « faire », les paraphrases utilisant les termes «premier » ou «d'abord» deviennent malaisées, et c'est bien le terme commencement lui-même qui s'impose : « être / entrer dans son commencement »...

\section{Le paradoxe de l' « existence partielle »}

Soit donc le commencement, et la glose suivante également proposée dans le Petit Robert : «Existence partielle. Dr. commencement de preuve par écrit. »

Les deux volets de cette formulation constituent un paradoxe :

- d'un côté l' « existence », qui est affaire de tout ou rien (il y a une preuve ou il n'y en a pas, tout au plus peut-on douter de ce que tel ensemble d'indices suffit à constituer une preuve);

\footnotetext{
${ }^{4}$ Le même principe diachronique accorde la prééminence au tour transitif dans les articles de finir ( « conduire à son terme en faisant ce qui reste à faire »), toumer (" façonner au tour ; faire mouvoir »), travailler («vx. faire souffrir»), geler (« faire souffrir du froid»), rouler (" déplacer... en le faisant tourner sur lui-même»), etc., par opposition à pendre, monter, chanter, bouder. A la diachronie s'ajoute la tradition lexicographique, de sorte qu'on ne saurait interpréter cette présentation comme révélatrice d'une quelconque intuition des sujets parlants, n'était une tendance générale de privilégier les activités des agents humains.
} 
- d'un autre côté "partielle», qui introduit une mesure, celle sous-jacente à toute présentation de commencer comme distinguant un premier point au sein d'une série.

Une façon, toute provisoire et insuffisante, de synthétiser cette définition paradoxale consiste à dire que le commencement a trait à la modalité d'existence / manifestation d'une entité. Cette caractérisation minimale permet déjà de lever le voile sur certains faits relevés dans la littérature consacrée au verbe commencer.

Pourquoi donc Le voisin commence le couloir ne peut-il pas désigner la partie initiale d'un déplacement linéaire du sujet? Une fois admis qu'il s'agit du mode d'existence du terme en position de complément (le couloir), on s'explique que l'endroit où se trouve le voisin n'est pas en cause. Ne seront donc licites que les interprétations affectant en propre le référent du SN le couloir, quel qu'il soit (il peut s'agir d'une image de couloir) : production, transformation ou reproduction - disons: construire, tapisser/laver, dessiner etc. -, à l'exclusion de ce qui concerne l'agent. En revanche, on dira sans peine: A peine eut-il commencé de traverser le couloir que l'alarme retentit. Ce que décrit la structure auxiliée n'est plus ce qu'il advient du couloir, mais concerne le procès traverser le couloir prédiqué de il: un événement que l'on dit perturbé dans son déroulement par l'alarme, donc pas tout à fait l'événement qu'on attendait. Un commencement d'événement, et non plus un commencement de couloir.

D'une façon comparable s'explique que commencer une symphonie se dise des interprètes (qu'ils jouent la symphonie actuellement ou entament les séances de répétition), et non de l'auditoire: ce dernier n'a pas prise sur l'existence de la symphonie. Et si l'on peut dire commencer le Chapitre IV du Capital d'un lecteur, c'est parce qu'un texte nécessite un lecteur pour être déchiffré - le lecteur est du côté de l'interprète engagé dans un processus de reproduction, plutôt que de celui de l'auditoire d'une symphonie ou du public d'un film ${ }^{5}$. Enfin, en l'absence même de toute interprétation agentive, dans Jacques commence une bonne grippe, la grippe n'a pas d'existence sans Jacques qui constitue son support; et dans Le 12 de la Rue de la Gare commence la Rue Pierre Girard, la Rue Pierre Girard ne serait assurément pas ce qu'elle est sans le 12 Rue de la Gare.

La piste de l' «existence / manifestation partielle » affranchit de la coûteuse nécessité de rétablir dans chaque énoncé quelque prédicat enfoui (thèse de l'ellipse) ou de conférer une dimension processive à une entité qui, comme le référent de couloir, en est a priori dépourvue (thèse de la «coerc[it]ion de type »). Elle repose toutefois, répétons-le, sur un paradoxe. En outre, elle se heurte apparemment à un écueil : le caractère fortement contraint des emplois intransitifs. La section qui suit leur est consacrée.

\section{Comment commencer est-il possible?}

Observons les conditions d'émergence du lexème commencer à partir de manipulation expérimentales de la séquence la plus brève possible (donc : intransitive).

\footnotetext{
${ }^{5}$ A moins d'envisager que l'on «visionne » une cassette vidéo (je commence le film est bien attesté dans ce sens sur l'Internet).
} 


\section{1. ?? Le chapitre IV commence}

La séquence ?? Le chapitre IV commence paraît fort improbable, difficilement interprétable, instable ; elle exige certains aménagements :

- introduction d'un SN processif :

La rédaction du chapitre IV commence.

- ajout de circonstanciels :

Le chapitre IV commence ici.

Le chapitre IV commence bien / par une coquille.

- transitivation :

Ce passage célèbre commence le chapitre IV.

Je commence le chapitre $I V$.

Ces manipulations qui assurent la bonne formation de la séquence sont autant d'indices sur le fonctionnement du verbe commencer. Elles peuvent être exploitées de deux façons: en tant qu'elles «sauvent» (rendent possible) l'emploi du verbe et constituent les conditions sine qua non de son apparition dans un texte; mais aussi, comme on le montrera plus loin, dans la mesure où elles permettent de dégager ce qui, dans commencer, est sujet à variation indépendamment des structures syntaxiques.

Sans doute y a-t-il quelque artifice à rapprocher ces structures par ailleurs si différentes. A noter en particulier que certains sauvetages sont combinables entre eux, alors que d'autres s'excluent mutuellement. Ainsi, l'ajout de bien associé à la transitivation produit la séquence Le chapitre IV commence bien le Livre II où l'adverbe perd la valeur d'évaluation qui lui était associée dans la structure intransitive (comparer : Le chapitre IV commence très bien vs * Le chapitre IV commence très bien le Livre II). Avec la transitivation, bien n'est plus interprétable que dans le cadre d'une relation inter-énoncés : il porte alors sur l'ensemble de l'énoncé, non sur commencer, et acquiert une valeur de confirmation ${ }^{6}$.

Toutefois, par delà l'incontestable hétérogénéité des solutions de sauvetage proposées, celles-ci partagent un point commun : chacune d'elle fonde une double prise en compte du terme chapitre $I V$, à savoir :

- le chapitre IV tel qu'il se manifeste dans une portion de l'espace-temps ;

- le chapitre IV en tant qu'il excède cette manifestation spatio-temporelle.

Dans Ce passage célèbre commence le chapitre $I V$, les deux modes de prise en compte du terme en jeu sont lexicalisés: le sujet désigne nécessairement un fragment, un ingrédient du complément le chapitre IV (on n'a pas * La page 8 commence le chapitre IV). En d'autres termes, ce passage offre une saisie partielle du chapitre IV.

La transitivation agentive - Pierre commence le chapitre $I V$ - autorise également une double représentation de l'entité désignée par le complément: a) le chapitre IV appréhendé au travers d'une visée de l'agent (un chapitre accompli, achevé, qui serait donc tout entier présent) ; et b) ce qui du chapitre IV est effectivement manifesté hic et nunc, défini comme incomplet au regard de la visée.

\footnotetext{
${ }^{6}$ Confirmation simple ou argumentative, respectivement :

- Effectivement, le chapitre IV commence bien la deuxième section.

- Le Chapitre IV commence bien la Deuxième section, pourquoi ne pas imaginer que le chapitre XXV était, à l'origine, l'épilogue?

Il en irait de même avec Je commence bien le chapitre IV.
} 
Le simple ajout de bien, sans transitivation, effectue une division comparable via un jugement différentiel : dire que le chapitre commence bien, cela revient à envisager une suite dont l'évaluation est en suspens; du même coup, c'est l'évaluation du chapitre dans son intégralité qui est en question, une discordance étant toujours possible (le chapitre commençait si bien...).

L'adverbe ici (le chapitre IV commence ici), quant à lui, il pointe un lieu où le chapitre $I V$ se manifeste sans pour autant y être tout entier contenu. L'observation suivante concernant la nature des localisateurs spatio-temporels est triviale, mais essentielle : $\mathrm{La}$ 4ème partie commence page 8 / la semaine prochaine implique une partie excédant le localisateur qui lui est assigné : une partie qui compte plus d'une page, ou encore susceptible de durer plus d'une semaine (partie d'échecs).

Reste enfin la transformation du $\mathrm{SN}$ le chapitre $\mathrm{IV}$ en $\mathrm{SN}$ de nature processive : la rédaction du chapitre $I V$. Le recours au procès de rédaction rend possible le départ entre le chapitre $I V$ rédigé et sa rédaction, c'est-à-dire entre le produit et le processus dont il résulte. Ainsi se retrouve l'hiatus constitutif de commencer, un hiatus entre une complétude visée et une incomplétude de fait.

\subsection{Le commencement et la fin}

L'étude distributionnelle élémentaire qui précède permet de préciser le paradoxe de l'existence / manifestation partielle postulé à partir du nominal commencement: de façon inattendue, il est apparu que commencer mettait invariablement en jeu non pas seulement une suite du chapitre $I V$, mais celui-ci dans son intégralité. Le verbe commencer implique la prise en compte d'une fin, d'une totalité ou d'une plénitude indispensable à la définition, par défalque, de ce qui commence.

De là s'ensuivent des contraintes qui pourraient paraitre au premier abord purement aléatoires. Observons que malgré le caractère processif du déverbal compte, la séquence ??Le compte commence parait nettement moins naturelle que Le compte à rebours commence ou encore Le décompte commence. De fait, le commencement ne fait sens que relativement à une perspective, le «zéro » vers lequel tend le compte à rebours, ou encore l'ensemble circonscrit impliqué par le préfixé décompte (« décomposition d'une somme, d'un ensemble, en ses éléments de détails »-Petit Robert, 1989).

S'ensuivent également de nombreuses contraintes distributionnelles, lexicales et interprétatives pesant sur les compléments des diverses constructions transitives :

- le blocage du partitif dans la séquence * Jean a commencé $d u$ fromage, qu'il faut comparer avec Jean a pris du fromage: la quantité désignée par $d u$ fromage n'est pas rapportée à un tout, elle se définit de façon circulaire comme "Jean a pris la quantité de fromage qu'il a prise ».

- les filtrage interprétatifs et lexicaux que Kleiber (1999) résume en termes de «progression quantitative homogène»: commencer un dictionnaire implique que la quantité de procès visée par l'agent corresponde à la totalité du dictionnaire, qu'on soit rédacteur, illustrateur, relecteur d'une maison d'édition (mais certes pas l'utilisateur occasionnel). L'homogénété en question ici n'est autre que celle que confère la définition du tout.

Il est crucial d'ajouter que ce tout peut être mis en place contextuellement. Soit ??commencer un / le verre, incontestablement plus contraint que commencer une / la bouteille 
(Kleiber, op. cit.). L'explication de cette donnée de prime abord énigmatique repose sur les propriétés de la séquence le / un verre qui, contrairement à la / une bouteille, renvoie à une mesure: que l'on compare un grand verre - grand concerne la dimension ou la contenance - et une grande bouteille, qui s'interprète tendanciellement comme une bouteille de qualité exceptionnelle. Néanmoins le / un verre peut être défini comme une totalité dans les contextes interruptifs engendrés par à peine: A peine avait-il commencé le verre que...

Enfin, la totalité peut être définie à rebours, ce qu'illustre le cas du lexème tout lui-même. L'étrangeté de ??Tout a commencé (tel quel) repose sur une contradiction: l'inscription du tout dans le temps (ici par le truchement de l'accompli a commencé) bloque la dualité «partie» vs «tout» indispensable au fonctionnement de commencer. Ceci explique la nécessité d'une détermination différentielle telle que ici, un beau matin de mai, l'an dernier, à cause de ta soeur. Mais il existe un autre sauvetage possible : chacun pourra vérifier ou faire vérifier que Tout a recommencé améliore très sensiblement la séquence. Or l'introduction de re-relève d'une logique opposée à celle de ici, logique qui répond au mécanisme à «double détente» du préfixe re- (cf. la description minutieuse de ce préfixe dans Jalenques, 2000) : cette fois-ci, le tout inscrit dans le temps est, dans un deuxième temps, qualifié de déficient / partiel du point de vue d'une finalité nouvelle. D'où s'ensuit que Tout a recommencé ne signifie pas un deuxième commencement, mais un commencement faisant pièce à une première fin, et orienté vers une seconde.

\section{Propositions pour une forme schématique de commencer}

Pour formuler de façon synthétique les remarques qui précèdent, je dirai que ce qui commence (ou ce qui est commencè), se trouve caractérisé de façon paradoxale : c'est ce dont la fin $X$ ( $X$ en tant que tout achevé, accompli, parfait etc.) se trouve programmée au travers de sa manifestation xi dans le temps et / ou l'espace.

Dans la mesure où elle repose sur un paradoxe, cette formulation ne livre pas quelque noyau sémantique en langue. Elle constitue bien plutôt une première tentative de déployer les paramètres en jeu dans le fonctionnement contextualisé du lexème, et ne prendra tout son sens que lors de l'étude de la variation de l'unité. Toutefois, quelques éclaircissements s'imposent dès maintenant :

1) La manifestation xi mobilise un repère spatial (ça commence lä) ou temporel (ça commence maintenant). Ce qui commence se déroule pour ainsi dire «sous nos yeux»- un observateur-spectateur-témoin est requis. Tout énoncé comportant commencer implique nécessairement un quantum non quelconque d'espace-temps. Lorsqu'il n'est pas explicité, ce quantum est identifié au moment où l'on parle : il commence exclut toute interprétation statique ou de prédication de propriété (comparer avec il existe, il écrit) ${ }^{7}$.

Cet ancrage déictique de xi n'est a priori pas à même de fournir la stabilité qualitative définissant un tout : en $\mathbf{x i}, \mathbf{X}$ se donne sur le mode de l'incomplétude, de l'ébauche, du «à continuer». Que $\mathbf{X}$ ne se résorbe pas en $\mathbf{x i}$, cela distingue commencer

\footnotetext{
7 Sauf coup de force consistant à pérenniser l'instant du commencement, cf. le souhait de V. Hugo : «être ce qui commence ».
} 
de finir, mais aussi d'entamer. Le chapitre IV est fini, La baguette est entamée décrivent des états stabilisés (on parle parfois d' "état résultant») du chapitre IV et de la baguette : le chapitre fini n'est plus à faire, la baguette entamée est irrémédiablement tronquée. A l'inverse, Le chapitre IV / la baguette est commencé(e) sont la promesse d'une continuation et même, pour anticiper le point suivant, d'un achèvement en perspective.

2) De façon non contradictoire, la manifestation xi donne accès à la représentation de $\mathbf{X}$ comme un tout stabilisé. Ce qui commence tend, ipso facto et irréfragablement, vers une forme de stabilité. Un programme est lancé, inéluctable. Où l'on retrouve l'idée de premier point ou de seuil : il suffit qu'une manifestation xi soit construite pour que $\mathbf{X}$ devienne envisageable.

Cela revient également à dire que l'incomplétude (X ébauché, partiel) n'est pas première : elle s'obtient par étalonnage avec le tout que permet de viser xi (X complet, parfait, achevé, terminé).

3) La tension résidant entre $\mathbf{x i}$, manifestation partielle de $\mathbf{X}$, et la représentation de $\mathbf{X}$ comme un tout stabilisé, peut se définir comme un processus. Ainsi se comprennent les affinités de commencer avec des termes à sémantique processive, et des contraintes interprétatives comme celles qui pèsent sur des termes comme jour ou nuit : La nuit commence ne signifie pas la fin du jour, mais annonce une nuit blanche; de la même façon, Le jour commence ne met pas en jeu le même jour que Le jour commence à poindre. Dans ces deux cas, dans la construction non auxiliée, le jour (la nuit) désigne les activités dont le jour (ou la nuit) sera fait.

\section{Plans de variation}

\section{1. <Délimitation $>$, <partition $>$, < surgissement $>$}

Revenons à notre séquence mal formée et aux stratégies de sauvetage combinables entre elles. Il en va ainsi pour l'ajout de l'adverbe ici et la substitution d'un SN idoine. Croisons Le chapitre commence ici et Le match commence ; nous obtenons : Le match commence ici.

Or ces trois séquences sont hétérogènes. Ce sont tout d'abord deux valeurs bien distinctes qui se dégagent, résumées par des étiquettes entre chevrons (" $<>$ ") :

\section{$<$ Délimitation $>$ : Le chapitre IV commence ici.}

Le localisateur ici spécifie le repère spatio-temporel où le chapitre IV s'actualise. Cet énoncé appelle un contexte où ledit chapitre se confond avec les autres chapitres : on parle donc d'un un ouvrage ne distinguant pas les chapitres avec toute la netteté voulue, ou encore d'un chapitre qui tarde à entrer dans le vif du sujet. Compte tenu de cette indétermination première, la construction de xi a précisément pour effet de délimiter $\mathbf{X}$ de ce qui n'est pas $\mathbf{X}$. 


\section{<Partition > : Le match commence ici (et.... / mais...)}

L'ajout de ici suggère que le match peut, si les circonstances l'exigent, s'interrompre pour se poursuivre ailleurs $^{8}$ : entre $\mathbf{x i}$ (se déroulant ici) et $\mathbf{X}$ (se déroulant certes ici, mais peut-être aussi ailleurs) est maintenue une distance. La même distance était maintenue par l'ajout de bien dans Le chapitre IV commence bien : d'une part l'évaluation du commencement, d'autre part celle du tout.

\section{$<$ Surgissement $>$}

Pour faire apparaittre cette troisième valeur, il convient d'observer le contraste Le match commence vs Un (vrai) match commence:

Avec le prédéterminant le, la valeur obtenue est celle de $<$ délimitation $>$, ce qui justifie que cette séquence soit le plus souvent accompagnée d'un actualisateur : Puis / Enfin / Et... le match commence (narration) ; En place / Chut / Attention / Tiens... le match commence (en interlocution). La différence avec le chapitre réside dans le caractère événementiel de le match: le match contient en soi la coordonnée spatio-temporelle que ici apportait de l'extérieur au SN le chapitre.

Avec le prédéterminant un, ce qui advient se trouve eo ipso qualifié de « match». Cette valeur n'est donc pas celle de $<$ délimitation $>$, laquelle impliquerait que $\mathbf{X}$ fût déjà donné. Mais elle n'est pas non plus identifiable à la valeur de partition : $\mathbf{X}$ n'est pas divisé en phases. Il y a ici surgissement de $\mathbf{X}$ à la faveur du jugement du sujet énonciateur. C'est cette troisième valeur qui sera étiquetée $<$ surgissement $>$.

$<$ Délimitation $>$, < partition $>$ et $<$ surgissement $>$ sont trois façons de mettre en œuvre la relation $\mathbf{x i}-\mathbf{X}$ telle qu'elle a été caractérisée plus haut ; chacune repose sur la prééminence accordée à $\mathbf{x i}$ ou à $\mathbf{X}$, respectivement: $\mathbf{X}$ est donné a priori mais réenvisagé à partir de $\mathbf{x i}(<$ délimitation $>) ; \mathbf{X}$ et $\mathbf{x i}$ coexistent $(<$ partition $>$ ); $\mathbf{x i}$ introduit $\mathbf{X}$ (<surgissement $>$ ).

Ces trois valeurs reposent en partie sur les propriétés conférées au SN : ici s'opposent les $\mathrm{SN}$ «événementiels » ou "procéduraux » tels que le match, et ceux qui ne le sont pas ; ici également se joue la différence entre un et le (cf. Le match commence / Un (vrai) match commence). C'est donc le statut conféré au SN qui est en jeu.

\subsection{SN commence : l'exemple de Ça commence}

Je soutiens que les trois valeurs ci-dessus sont détachables des contextes qui ont permis de les discerner: les oppositions «présence» vs «absence d'un circonstant» et «prédéterminant le» vs «prédéterminant un». En réalité, elles ne disent pas des effets de sens figés dans un eden d'idées pures et inaltérables, mais trois registres de variation liés à trois façons de construire une manifestation xi (une « occurrence») de $\mathbf{X}$. La démonstration s'appuiera sur la séquence minimale Ça commence.

\footnotetext{
${ }^{8}$ Le point crucial est naturellement qu'un chapitre se conçoit d'un seul tenant, alors qu'un match, en sa qualité de terme procédural, n’est pas ancré au lieu où il se déroule.
} 
Quelques aspects de commencer

\section{Ça commence : <Délimitation >}

Tais-toi, ça commence!

Dépêche-toi, ça commence!

On observe dans ces énoncés une proximité avec, respectivement, c'est commencé ou ça va commencer.

Pour naître, les fatus sont obligés d'oublier ce qu'ils savent. Heureusement que j'avais pris des notes assez régulièrement. Les voici.

Attention, ça commence.

Tout d'un coup, il y eut une lumière folle.

Elle rayonna dans l'espace...

(François Weyergans, La vie d'un bébé, 1986, Frantext)

Cet énoncé est ambivalent. Suivant une première interprétation, la plus immédiate, c'est la relation de la naissance qui commence : ça commence pose une balise métatextuelle pointant la borne gauche des notes prises jadis. Mais il peut également s'agir de la naissance elle-même, dont le récit reproduirait fidèlement les péripéties. A moins que ces deux interprétations ne fassent corps.

Dans tous ces énoncés, $\mathbf{X}$ est en attente de l'actualisation dont $\mathbf{x i}$ vient fixer l'instant ou le lieu. Comme on l'a déjà signalé, ces énoncés appellent tendanciellement la présence d'un actualisateur : attention, tais-toi, dépếche-toi, etc., à moins qu'ils ne soient prononcés avec l'emphase qui caractérise certains énoncés performatifs: Le spectacle commence!, prononcé avec des intonations de bateleur, peut suffire à marquer l'entrée dans le spectacle.

Ce qui les distingue les uns des autres, autrement dit le jeu caractérisant ici la valeur de < délimitation>, c'est la façon dont se justifie l'actualité de xi. Cette actualité peut découler de la prise en compte du comportement effectif ou envisagé d'autrui (lequel est appelé à moduler en conséquence son comportement); elle peut être justifiée par l'acte même de profération, comme le suggère la seconde interprétation du texte chatoyant de Weyergans : ça commence lance le processus de la naissance, à la frontière entre le constatif et le performatif.

Du coup, xi ne s'interprète pas comme une ébauche de $\mathbf{X} ; \mathbf{X}$ se donne comme un tout homogène. De la même façon que Les blés commencent ici n'impliquent pas une qualité particulière des blés: même en bordure de champ, les blés sont (totalement) les blés.

De cet emploi relève également La pluie commence, qui se trouve généralement en contextes narratifs (on dira plus volontiers Tiens, il commence à plewvoir que Tiens, la pluie commence), et s'avère considérablement moins fréquente que La pluie commence à tomber?

\footnotetext{
${ }^{9}$ Voici les rares exemples glanés sur l'Internet à l'aide du moteur Google (alors que les exemples comportant l'infinitive sont très nombreux):

La journée s'annonçait ensoleillée mais voilà que la pluie commence et ne s'arrête plus.

Le match est sympa. Mais à 3/2, la pluie commence...

La route monte en lacets vers Soe, la pluie commence. L'unique essuie-glace $d u<$ minibus-taxis $>$ fait ce qu'il peut.
} 
Alors que dans cette dernière séquence le commencement peut correspondre à un degré (une pluie peut-être moins drue), la pluie qui commence (absolument) se présente en bloc: il ne s'agit plus de premières gouttes, mais d'un événement météorologique perturbateur (cf. les contextes des exemples de la note infrapaginale).

Prédication d'existence de xi et conformité qualitative à $\mathbf{X}$ peuvent être décrits, à la suite de S. de Vogüé (1999), en termes d'instanciation discrète (quantitative et qualitative) de la notion $\mathbf{X}$. Rappelons que dans cette classification, il est proposé d'associer l'instanciation discrète d'une notion au registre de l'Histoire, registre où ce ne sont pas les sujets mais les faits du monde qui valident les dires: ça s'impose comme une donnée du monde. D'où les affinités de cette classe d'emplois avec la catégorie de la performativité : Le spectacle commence! comme analogue de Je déclare la séance ouverte.

\section{Ça commence : <Partition $>$}

Les énoncés de ce groupe se caractérisent par leur dimension réactive (cf. les passages en caractères romains ci-dessous) :

- (...) je laisse ça aux jeunes.

- Ob! Vous n'êtes pas encore bien vieux.

- Dame! Ca commence. Chacun son tour.

(Jules Renard, Journal, 1910, Frantext)

"Ça n'est pas fini, grogna-t-il en marchant. Ca commence, ça commence » Les épaules en avant, il avançait comme un haleur vers un pays confus dont il savait seulement qu'on y tuait...

(André Malraux, La condition bumaine, 1933, Frantext)

- C'est elle qui reçoit don José?

- Oui.

- Il l'aime?

- Ca commence.

- Bon; mais pourquoi le reçoit-elle ici et non rue de Castiglione? (Pierre-Alexis Ponson du Terrail, Rocambole, 1859, Frantext)

La valeur de <partition> est liée à un contraste, dont l'interprétation varie suivant le mode de construction de $\mathbf{x i}$ :

- «plus tôt que tu ne le penses » - qui est de l'ordre du déjà ;

- « cela n'est pas fini : cela ne fait que commencer»-de l'ordre du encore;

- «c'est en bonne voie »- de l'ordre du presque.

L'enjeu de ces énoncés est l'hiatus maintenu entre d'une part $\mathbf{X}$ introduit contextuellement et d'autre part xi. La manifestation actualisée xi permet, respectivement :

- de reprendre $\mathbf{X}$, au sens où l'on «reprend quelqu'un » : l'interprétation du type déjà est polémique, l'interlocuteur suggérant que je ne suis pas vieux;

- de redire $\mathbf{X}$ : dans le second exemple (citation de Malraux), encore marque une insistance, d'ailleurs doublée iconiquement par l'itération : « şa commence, ça commence »; 
- de reformuler $\mathbf{X}$ : l'interprétation du type presque n'infirme ni n'insiste - elle marque une précision.

La différence avec le cas précédent est qu'il n'y pas nécessairement conformité qualitative entre xi et $\mathbf{X}$ : xi n'est qu'un début, une esquisse, un premier temps s'opposant à la plénitude notionnelle de $\mathbf{X}$. Sous cet aspect, l'instanciation de la notion $\mathbf{X}$ par xi mobilise donc fondamentalement une dimension quantitative (xi n'instancie pas $\mathbf{X}$ qualitativement), ce qui correspond à un fonctionnement désigné « dense » dans la classification mentionnée plus haut.

\section{Ça commence : < Surgissement >}

- Dis, papa, quand est-ce qu'on arrive?

- Allons bon, il ne manquait plus que ça. Ça commence... Nous venons à peine de prendre la route!

(...une bonne) cinquantaine de kilomètres, beaucoup de côtes, ils commencent à avoir les genoux qui tremblent, les mollets et les cuisses qui brûlent. A la sortie du village, la scène! Ca commence. Quatre gendarmes et leur brigadier lèvent les bras, alignés, le dos au mur de la gendarmerie. Une douzaine de maquisards leur ôtent pistolets, étuis, ceinturons...

(Jean-Pierre Chabrol, La folie des miens, 1977, Frantext).

Cette fois-ci, c'est à partir de $\mathbf{x i}$ que $\mathbf{X}$ acquiert une visibilité. Un élément du contexte (la question impatiente de l'enfant, la scène) est reconnu par le sujet énonciateur comme prodrome d'un $\mathbf{X}$ qui n'est pas nommé. Ce qui se déroule effectivement peut certes être détaillé, comme dans le récit de J.-P. Chabrol; mais même dans ce cas, le terme ça ne saurait être interprété comme le pur et simple substitut d'une terme présent dans le contexte. En somme, $\mathbf{X}$ ne se donne que sous l'espèce de $\mathbf{x i}$, ce qui débouche sur une déqualification de $\mathbf{X}$. En contexte interlocutif, ça commence se rapproche ici de « (ah non,) ça ne va pas (encore) recommencer! $»^{10}$.

C'est donc ici par le truchement d'un sujet qu'un $\mathbf{X}$ absent du texte se trouve néanmoins évalué sur le plan qualitatif. On parlera d'instanciation compacte par xi de la notion $\mathbf{X}$, laquelle instanciation implique le jugement d'un sujet et relève à ce titre du registre du discours.

\subsection{Modes de contextualisation}

Les trois valeurs décrites ci-dessus entretiennent des affinités avec certains SN dans la structure intransitive, par exemple: le match <délimitation>, les ennuis <surgissement>. J'appellerai «modes de contextualisation» le plan de variation lié à l'existence éventuelle de «relais » de la forme schématique dans l'entourage lexical et

\footnotetext{
${ }^{10}$ Les traductions russes confirment la particularité de cette classe d'emploi. «Ça commence» dans ses valeurs < délimitation> et < partition> pourra tantôt se traduire Načinaetsja (verbe imperfectif au présent), tantôt Načalos' (verbe perfectif au passé), alors que les emplois de type <surgissement> ne peuvent se traduire qu'avec l'imperfectif présent: Načinaetsja! En effet, le perfectif passé načalos' implique que $\mathbf{X}$ soit donné indépendamment de xi (envisagé, attendu, redouté etc.), or ici il s'agit d'identifier $\mathbf{X}$ à partir de $\mathbf{x i}$ n'est donc pas donné préalablement.
} 
grammatical de commencer ${ }^{11}$. Les modes de contextualisation constituent un plan de variation à part, qui se combine tant avec les trois modes d'instanciation de $\mathbf{X}$ qu'avec la variation syntaxique. Dans le cas de commencer, la question essentielle est de savoir dans quelle mesure les entourages du lexème commencer définissent $\mathbf{X}$ indépendamment de la prédication d'existence de xi, d'où l'importance primordiale du SN désignant $\mathbf{X}$. On observe des analogies avec la tripartition ci-dessus, mais il n'y pas véritable coïncidence :

1) Commencer entérine le mode de construction de $\mathbf{X}$ induit du $\mathrm{SN}$ (sujet ou complément d'objet) lui-même. $\mathbf{X}$ est en soi envisagé relativement à un point de stabilisation : Le match commence, L'attente commence. Ce qui distingue ces séquences est le caractère respectivement programmé (le match) ou incident (l'attente) de la fin.

Les compléments d'objet définissant une mesure de procès ressortissent également à ce cas : commencer une bouteille; commencer un cheval (à distinguer de commencer le cheval pour « se mettre à l'équitation », cf. infra).

Enfin, citons les cas où commencer organise une relation extrémité / tout donnée indépendamment :

Trois barreaux rouillés commençaient une grille de fer large et basse. (Frantext)

Le 12 de la Rue de la Gare commence la Rue Pierre Girard.

2) La problématique de la stabilisation de $\mathbf{X}$ (X envisagé comme un tout) repose exclusivement sur la prise en compte d'un agent.

A côté de Le match commence (catégorie précédente), on a plus difficilement je commence un / le match, à moins d'être un participant du match, lequel participant peut se retirer du jeu à son gré : Quand je commence un match, je ne sais jamais si je vais le finir. La fin est alors non plus celle inscrite dans la sémantique du SN le match, mais une simple cessation reposant sur le bon vouloir de l'agent et non nécessairement visée a priori.

De la même façon, Je commence l'hébreu, le cheval, la Nivaquine, une cure etc.

3) $\mathbf{X}$ pris comme un tout n'a pas de support en dehors de la prédication de $\mathbf{x i}$ effectuée par commencer. Dans ce cas, la stabilisation ne coïncide plus nécessairement avec l'atteinte effective d'une fin : Les ennuis commencent.

De même :

Oui, la guerre était finie (...) ce soir c'était une vraie fête ; la paix commençait, tout recommençait. (S. de Beauvoir)

Tout annonce que mes affaires à l'Intérieur vont enfin se terminer, et que mon bonheur va commencer.

(V. Hugo)

Nulle catastrophe finale ne se laisse pressentir dans ces commencements. La plénitude est ici présente en contrepoint au contraste temporel: une paix et un

\footnotetext{
${ }^{11}$ Sur ce point, on se reportera avec profit à l'article Paillard (2000).
} 
bonheur qui n'alterneraient pas avec la guerre et le malheur, une paix et un bonheur sans ombre. On retrouve ici la dynamique d'attraction vers un climax observée par Franckel (1989) au sujet de Je commence à être (vraiment, totalement, complètement) fatigué.

De ce type relève également Pierre commence une maladie, une fluxion de poitrine, une grippe, une dépression, un cancer du colon, une maladie d'Alžbeimer etc. Seul l'indéfini un est possible, on a donc exclusivement Pierre commence une / *la grippe en face de Pierre a une / la grippe. De plus l'affection doit être évolutive, cf. : Pierre a / * commence un virus, un souffle au coeur, une insuffisance rénale. Esquissée à grands traits, l'explication de ces faits peut se donner en partie dans le cadre du mode de variation mis en jeu ici : d'une part, la maladie ne préexiste pas à sa manifestation chez le sujet (cf. l'indéfini); d'autre part, et c'est ici le lien avec ce troisième type, le $\mathbf{X}$ auquel est rapporté la manifestation $\mathbf{x i}$ n'a d'autre support que le diagnostic d'un sujet distinct du localisateur, lequel sujet décèle la maladie au travers de ses prodromes et, éventuellement, l'évalue : commencer une méchante grippe, une grave maladie, une dépression sérieuse etc.

À ce type ressortissent enfin les exemples où ce qui commence est en soi un commencement (qui ne saurait donc donner à soi-même la perspective de sa propre fin), ce qu'illustre l'exemple suivant:

[...] Cet enfant, je pense qu'il existe, qu'il commence, qu'il a une vie toute newve à vivre.

(R. Martin du Gard)

Ici, le commencement ne marque pas un stade dans l'existence de l'enfant (l'enfance par rapport à la sortie de l'enfance), mais ne vaut qu'au titre d'ouverture progressive à la plénitude de la vie, comme le confirme la suite «une vie toute neuve à vivre».

\section{Remarques en guise de conclusion}

Les remarques qui suivent ébauchent des prolongements possibles de la présente recherche, tant au sujet du lexème commencer, qu'à celui d'un éventuel contrecoup pour une théorie qui chercherait à articuler sémantique et syntaxe dans l'étude du lexique verbal français.

\subsection{Le statut $d u$ Co et la question des «constructions absolues»}

Appelons Co le premier terme de la relation prédicative, pour le distinguer du terme plus large de «sujet syntaxique » désignant tout constituant lié au syntagme verbal au travers du mécanisme d'accord (y compris $i l$ dans $I l$ commence à pleuvoir ou Il se commence beaucoup d'babilitations ces temps-ci).

Les configurations syntaxiques se distinguent suivant que le Co représente $\mathbf{X}$ (Le chapitre IV commence ici), $\mathbf{x i}$ (Trois barreaux rouillés commencaient une grille de fer large et basse), $\mathbf{X}$ et xi (Le match commence) ou encore ni $\mathbf{X}$ ni xi. Ce dernier cas correspond notamment aux cas de figure listés sans commentaires ci-après :

- le Co représente un localisateur : Dominique commence une grippe ; 
- le Co représente un causateur: Dominique commence les prés $i c 1^{12}$ (Dominique fait en sorte que les prés commencent ici); (un commandant aux officiers) il faut que vous commenciez la retraite (faire en sorte que la retraite commence $)^{13}$;

- le Co représente un agent: Nous commençons la bouteille / le repas (entamer la bouteille, le repas $)^{14}$.

Tout à fait à part se trouve l'emploi que les dictionnaires qualifient d'« absolu », qui exige un sujet humain ou assimilé : Monsieur l'abbé commence. Un relevé exhaustif de «Co (animé) commence » sur Frantext fait apparaître les valeurs suivantes :

a. - «C’est Monsieur l'abbé qui commence » (attribution de tours de rôle dans un jeu);

b. - Didascalie (séquence introduisant ou ponctuant une prise de parole attribuée à l'abbé) ;

c. - Un commentaire agacé (proche de Monsieur l'abbé recommence, Monsieur l'abbé remet ça).

Aucun de ces emplois ne se prête à une analyse en terme d'ellipse lexicale (au sens de Cl. Blanche-Benveniste, cf. supra) : il serait malaisé d'insérer un constituant de la forme $\grave{a}+$ Infinitif. Dans tous ces cas, Monsieur l'abbé désigne en même temps un agent, et un terme associable à la forme schématique de commencer, respectivement :

a' - Monsieur l'abbé est identifiable à xi (il est le premier dans une série) ;

b' - Monsieur l'abbé correspond à $\mathbf{X}$ (Monsieur l'abbé est identifié à son vouloir dire : il est crucial que les paroles citées accompagnant cette didascalie ne constituent pas un tout et correspondent seulement aux premiers mots);

c' - Ici coexistent Monsieur l'abbé tel qu'il se manifeste habituellement (X) et sa manifestation actualisée (xi). Cette troisième valeur coïncide avec la valeur $<$ surgissement $>$ de ça commence.

En résumé, cet examen beaucoup trop rapide des configurations syntaxiques suggère que chacune d'entre elles doit être réenvisagée à la lumière des trois modes de

12 Continués par des saignées en éventail, aux endroits où la côte s'accuse, où je veux finir les emblavures et commencer les prés, ils [les fossés] arroseront mon herbe. (J. de Pesquidoux, Frantext).

13 Exemple forgé à partir de l'extrait suivant, ou le commandant suggère à ses officiers de donner l'artillerie contre ses propres troupes pour que leur retraite soit non pas la débâcle qu'elle est ( $L a$ retraite commence... Beaucoup se sont déjà sauvés), mais un repli stratégique maîtrisé (il faut commencer la retraite) :

- La route de Dinan est-elle libre?

- Je le crois.

- Il faut commencer la retraite.

- Elle commence. Beaucoup se sont déjà sauvés.

- Il ne faut pas se sauver; il faut se retirer. Pourquoi ne vous servez-vous pas de l'artillerie? (V. Hugo, Frantext)

${ }^{14}$ Il faudrait s'interroger sur les liens qui apparaissent entre l'opposition « causatif » vs « agentif » et les modes de construction du SN évoqués en I.1. : commencer le chapitre IV compris comme « faire accéder à l'existence (rédiger...) » vs commencer le chapitre IV au sens d'un travail interprétatif (lire...). 
fonctionnement définis plus haut. La question des éventuelles passerelles entre ces niveaux d'analyse distincts reste ouverte. Par exemple, il faudrait comprendre pourquoi le cas c' où le $C_{o}$ correspond à $\mathbf{X}$ et $\mathbf{x i}$, s'avère coïncider avec la valeur $<$ surgissement> de ça commence.

\subsection{Syntaxe et scénarios : la question des inférences}

Nous voici revenus à cette question emblématique des études sur commencer, celle des inférences licites à partir de commencer un livre où se noue aspect, type de procès et syntaxe (question de l'ellipse) mais aussi détermination du SN (mode de construction). S'agit-il de lecture ou de rédaction (hors contexte), de massicotage, illustration ou époussetage (si le contexte s'y prête) ou même de quelque autre activité plus ou moins incongrue qu'un conte rendrait possible, en mettant par exemple en scène un ogre bibliophage attablé.

Une fois les attendus proprement grammaticaux dégagés, la question peut paraitre oiseuse. Elle a pourtant un aspect linguistique : existe-t-il quelque propriété en langue qui prédisposerait certains agencements de formes plutôt que d'autres à mobiliser autre chose que la pure raison linguistique, disons le «bon sens» et les « connaissances encyclopédiques»? Car tel est bien le cas de commencer: la recherche d'un correspondant mondain au commencement est en général associée implicitement au plan de variation syntaxique.

En effet, ne sont mentionnés dans la littérature consacrée à ce point que certains emplois de la configuration Co-V-C1. Il s'agira donc de Dominique commence un livre, le cheval (dessiner, pratiquer, consommer, servir, dresser ${ }^{15}$ ), le linge (laver, repasser, repriser, teindre) etc. à l'exclusion de une grippe. Il existe ainsi des configurations dans lesquelles le SN se trouve immédiatement associé à ce qu'Abelson et Shank appelaient des scénarios (scripts) : la séquence un livre, si elle implique un référent préexistant, suscite la représentation du scénario <lecture> qu'il est loisible de détailler en sousséquences (ouvrir la couverture, regarder, tourner les pages les unes après les autres, etc.).

Ce moment où le linguiste se trouve contraint à décrire le monde correspond ici très précisément au cas de figure identifié plus haut dans la rubrique « instanciation discrète (quantitative et qualitative) de $\mathbf{X}$ », qui ressortit au registre de l'Histoire (cf. De Vogüé 1999 ; Camus 2002) : c'est dans ce cas que l'hiatus résidant entre $\mathbf{X}$ stabilisé et sa manifestation xi se dit sous la forme d'un processus ad hoc.

Si cette hypothèse se confirme, cela signifie que la question des inférences licites fut, sous l'influence des hypothèses fondées sur l'ellipse, illégitimement restreinte aux constructions transitives strictes. Et il semble bien que les constructions

\footnotetext{
${ }^{15}$ On notera que cet emploi désignant l'intervention d'un premier agent dans un processus de dressage, formation etc. est souvent accompagnée de la mention «vieilli ». Or ce que montrent les substantifs relevés par les différents dictionnaires, c'est que le caractère plus ou moins vieilli est fonction des représentations collectives et pratiques sociales correspondantes. Dans commencer un élève, ce qui paraît vieillot en l'occurrence, c'est plutôt la conception sous-jacente de l'éducation scolaire (l'élève ne devant son existence d'élève qu'à la succession de ses maittres, à l'instar de ces nourrissons que leur première nourrice commence). Cf. aussi l'univers que présuppose commencer une femme, qui est celui que dénonçait Kate Milett.
} 
dites «absolues » génèrent elles aussi des inférences, du moins dans cet emploi où L'abbé commence assigne des tours de rôles et se trouve compatible avec une infinité d'activités. Il en irait également de même dans la configuration discrète des emplois intransitifs : l'ajustement entre la manifestation xi et la totalité hors espace-temps $\mathbf{X}$ autorise un jeu exploité à merveille par cette publicité de voyagiste :

Les vacances commencent dans nos bureaux.

(Internet)

À chacun d'imaginer ce qui peut bien se passer réellement dans nos bureaux, ce que peuvent être ces bureaux où, de manière incongrue, commencent les vacances (de manière incongrue parce qu'il se trouve que les vacances sont précisémernt l'opportunité attendue pour s'échapper des bureaux); à chacun d'imaginer aussi ce que, par retour, il faut entendre par les vacances commencées de la sorte.

\subsection{Ni métaphore, ni métonymie}

À la métaphore et la métonymie, tropes envisagés successivement par Kleiber (1999) pour caractériser les modèles de construction du sens intégrant commencer, je propose de substituer un trope caractérisant en propre commencer. Ce trope se profile dans l'ébauche de forme schématique proposée : ce qui commence est ce dont la fin (X dans sa totalité : achevé, accompli, parfait etc.) se trouve programmée au travers de sa manifestation xi dans le temps et / ou l'espace. Ce trope qui consiste à dire le tout (X achevé, parfait...) par le menu (une manifestation xi) n'est autre que la synecdoque. D'où les figures du premier point, de l'accès, du seuil, mais aussi de l'inachèvement, de l'incomplétude.

Figures qui s'acharnent sur nos descriptions de commencer elles-mêmes, suivant cette exorbitante efficace qu'exercent les mots sur les théories qu'on en fait (cf. la thématique de l'ellipse). Le présent article, on l'a compris, ne prétend pas se soustraire à cette implacable mimésis ${ }^{16}$.

\footnotetext{
${ }^{16}$ Merci à S. de Vogüé, J.-J. Franckel, D. Leeman, D. Paillard, F. Thuillier pour leurs relectures critiques.
} 


\section{RÉFÉRENCES BIBLIOGRAPHIQUES}

ABELSON, Robert P., SHANK, Roger C., 1977, Scripts, Plans, Goals and Understanding (An Inquiry into Human Knowledge Structures). Hillsdale (New Jersey): Lawrence Erlbaum Associates.

APRESJAN, Jurij Derenikovič, 1974, Leksičeskaja semantika (sinonimičeskie sredstva jasyka) [Sémantique lexicale (les ressources synonymiques de la langue)]. Moscou : Nauka.

BLANCHE-BENVENISTE, Claire, 1975, "De l'ellipse », Cahiers de linguisitique, d'orientalisme et de slavistique, 5-6 : 31-41 [tiré à part amendé par l'auteur].

CAMUS, Rémi, 2002, «Le phénomène Borodino ». Caen : Cabiers de la MRSH, 31 : 65-76.

DE VOGÜÉ, Sarah, 1999, «Construction d’une valeur référentielle : entités, qualités, figure », Cerlico : la référence $2:$ Statut et processus, 12, Presses universitaires de Rennes : 77-106

FRANCKEL, Jean-Jacques, 1989, Étude de quelques marqueurs aspectuels du français. Genève-Paris : Droz.

GODARD, Danièle \& JAYEZ, Jacques, 1993, « Le traitement lexical de la coercion », Cabiers de linguistique française, $14: 123-149$.

JALENQUES, Pierre, 2000, Contribution à l'étude du préfixe RE- en français contemporain: pour une analyse compositionnelle du verbe regarder. Paris : Université Paris 7 - Denis Diderot, Thèse de doctorat en linguistique théorique et formelle.

KLEIBER, Georges, 1999, Problèmes de sémantique. La polysémie en questions. Villeneuve d'Ascq : Presses universitaires du Septentrion.

L.I.V. = RIX, Helmut (réd.), 2001, Lexikon der indogermanischen Verben (die Wurzeln und ibre Primärstammbildungen). Wiesbaden : Dr. Ludwig Reichert Verlag.

OŠANIN, I.M. (réd.), 1983, Bol'šoj kitajsko-russkij slovar' (v 4-x tomax) [Grand dictionnaire chinoisrusse (en 4 volumes)]. Moscou : Nauka.

PAILLARD, Denis, 2000, «A propos des verbes polysémiques: identité sémantique et principes de variation ", Syntaxe \& sémantique, n² $2: 99-120$.

PEETERS, Bert, 1993, "Commencer et se mettre à. Une description axiologico-conceptuelle», Langue française, $98: 24-47$.

PEETERS, Bert, 2002, «Les constructions du type commencer un livre : état de la question et nouvelles perspectives ». In LAGORGETTEE, Dominique, LARRIVÉE, Pierre (éd.), Représentations du sens linguistique. Munich : Lincom Europa : 167-186.

PEETERS, Bert, 2003, «Commencer à + infinitif : les leçons de la métonymie intégrée et de la piste métaphorique ». Louvain: Katholieke Universiteit Leuven (Preprint nr. 203 [2003]). 